\title{
Luminescent PLGA Nanoparticles for Delivery of Darunavir to the Brain and Inhibition of Matrix Metalloproteinase-9, Relevant Therapeutic Target of HIV-Associated Neurological Disorders
}

Tiziana Latronico ${ }^{1 \dagger}$, Federica Rizzi ${ }^{2,3 \ddagger}$, Annamaria Panniello ${ }^{3}$, Valentino Laquintana ${ }^{4}$, Ilaria Arduino $^{4}$, Nunzio Denora ${ }^{4}$, Elisabetta Fanizza ${ }^{2,3}$, Serafina Milella ${ }^{1}$, Claudio. M. Mastroianni ${ }^{5}$, Marinella Striccoli ${ }^{3}$, M. Lucia Curri ${ }^{2,3}$, Grazia M. Liuzzi ${ }^{*}$, Nicoletta Depalo ${ }^{3 *}$

${ }^{1}$ Department of Biosciences, Biotechnology and Biopharmaceutics, University of Bari, Via Orabona 4, 70126 Bari, Italy

${ }^{2}$ Department of Chemistry, University of Bari, Via Orabona 4, 70126 Bari, Italy

${ }^{3}$ Institute for Chemical and Physical Processes (IPCF)-CNR SS Bari, Via Orabona 4, 70126 Bari, Italy

${ }^{4}$ Department of Pharmacy - Pharmaceutical Sciences, University of Bari, Via Orabona 4, 70126 Bari, Italy

${ }^{5}$ Department of Public Health and Infectious Diseases, 'Sapienza' University, AOU Policlinico Umberto 1, 00185 Rome, Italy

*Co-Corresponding Authors: Grazia M. Liuzzi, e-mail: graziamaria.liuzzi@uniba.it.; Nicoletta Depalo, e-mail: n.depalo@ba.ipcf.cnr.it

$\$$ These authors contributed equally

Table 1: Absolute QYs (\%) of C-Dots in $\mathrm{CHCl}_{3}$ dispersion and C-Dots/DRV/PLGA nano-formulations, re-dispersed in $\mathrm{CHCl}_{3}$, according to the procedure described in the experimental section, as a function of the excitation wavelength.
Exc@380nm
Exc@400nm
Exc@410nm
Exc@420nm

\begin{tabular}{c|cccc}
\hline \hline C-Dots & 24 & 33 & 36 & 30 \\
C-Dots/PLGA/DRV & 20 & 23 & 27 & 22
\end{tabular}

